\title{
Palliative treatment of the advanced gastric cancer by means of surgery and HIPEC
}

\author{
Hoskovec $\mathrm{D}^{1}$, Varga $\mathrm{J}^{2}$, Antos $\mathrm{F}^{2}$, Kaspar $\mathrm{M}^{3}$, Vitek $\mathrm{P}^{4}$, Benkova $\mathrm{K}^{5}$, Dytrych $\mathrm{P}^{1}$, Konecna $\mathrm{E}^{2}$ \\ 1st Department of Surgery General University Hospital and 1st Medical Faculty Charles University, Prague, \\ Czech Republic. david.hoskovec@vfn.cz
}

\begin{abstract}
Objectives: We demonstrate a case report of the patient who suffered from advanced gastric cancer and was treated by means of surgery and HIPEC.

Background: Gastric cancer is a therapeutic challenge in the European countries due to late diagnosis, advanced stages of the disease in time of diagnosis and early recurrence in cases where a radical surgery is possible. Method: The patient with an advanced gastric cancer (pT3N2M1 - peritoneal and ovarian metastases) was treated by means of radical surgery in combination with hyperthermic intraoperative intraperitoneal chemotherapy (HIPEC) and early postoperative intraperitoneal chemotherapy (EPIC). Surgical treatment was followed by a standard chemotherapy. Due to recurrence, there was the second look surgery one year later again with HIPEC procedure.

Results: Patient survived 32 months after diagnosis and despite intraperitoneal recurrence she never developed clinically significant ascites and she never suffered from intestinal obstruction.

Conclusion: This case demonstrate a potential benefit of new oncosurgical approach -radical surgery + HIPEC

+ EPIC in the treatment of gastric cancers (Tab. 2, Fig. 6, Graph 4, Ref. 18). Text in PDF www.elis.sk.

Key words: gastric cancer, HIPEC, EPIC, radical surgery, peritoneal cytology
\end{abstract}

Gastric cancer is one of the most frequent malignant diseases worldwide even despite its decreasing incidence. Even though the incidence is highest in the East Asia countries, there were 192000 new diagnosed gastric cancer and 158000 deaths due to gastric cancer in Europe in $2000(1,2)$. The 5-year survival in the Europe is about $20 \%$ (1). The epidemiologic situation in the Czech Republic is well documented by the National cancer control program and is accessible online on www.svod.cz (Graph 1 and 2). The decreasing incidence but still a high percentage of advanced or unspecified disease in time of diagnosis is clearly visible (3).

Radical surgical resection is contemporary the only cure what gives the chance for longer survival despite new endoscopic treatment of early gastric cancer published in Asia countries (4) and new development in adjuvant and neo adjuvant treatment. There are many discussions about the extent of the resection (partial or total gastrectomy), extent of the lymphadenectomy (D1-3), presence of splenectomy, type of the reconstruction etc. All authors

${ }^{1} 1$ st Department of Surgery General University Hospital and 1st Medical Faculty Charles University, Prague, ${ }^{2}$ Department of Surgery University Hospital Bulovka and 1st Medical Faculty Charles University, Prague, ${ }^{3}$ Department of Radiodiagnostics University Hospital Bulovka and 1st Medical Faculty Charles University, Prague, ${ }^{4}$ Institute of Radiation Oncology University Hospital Bulovka and 1st Medical Faculty Charles University, Prague, and ${ }^{5}$ Department of Pathology University Hospital Bulovka, Prague, Czech Republic

Address for correspondence: D. Hoskovec, MD, 1st Department of Surgery, General University Hospital, U Nemocnice 2, CZ-120 00 Prague 2, Czech Republic.

Phone: +420.603147301 emphasize that the only radical treatment is $\mathrm{R} 0$ resection - there are not cancer cells left. However, can we reach R0 resection in cases with serosal infiltration (T3) or massive lymphatic spread of the tumour (N2 or 3)? There is a risk of undetectable micrometastases or free cancer cells in the peritoneal cavity or lymphatic system (minimal residual disease). Also, there are no satisfactory treatment guidelines for the stage IV $(4,5)$. In this stage, the survival is only several months (6).

\section{Case report}

We would like to demonstrate the potential of hyperthermic intraperitoneal intraoperative chemotherapy (HIPEC) in the treatment of advanced gastric cancer.

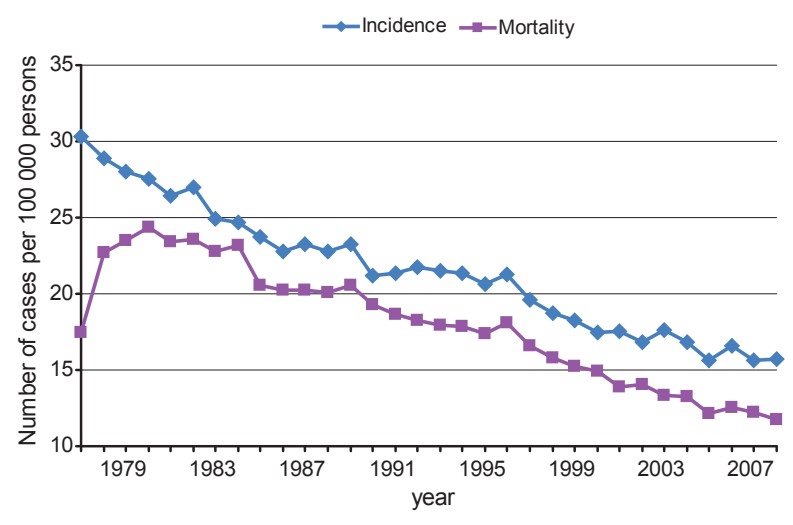

Graph 1. Gastric cancer incidence and mortality in the Czech Republic. 


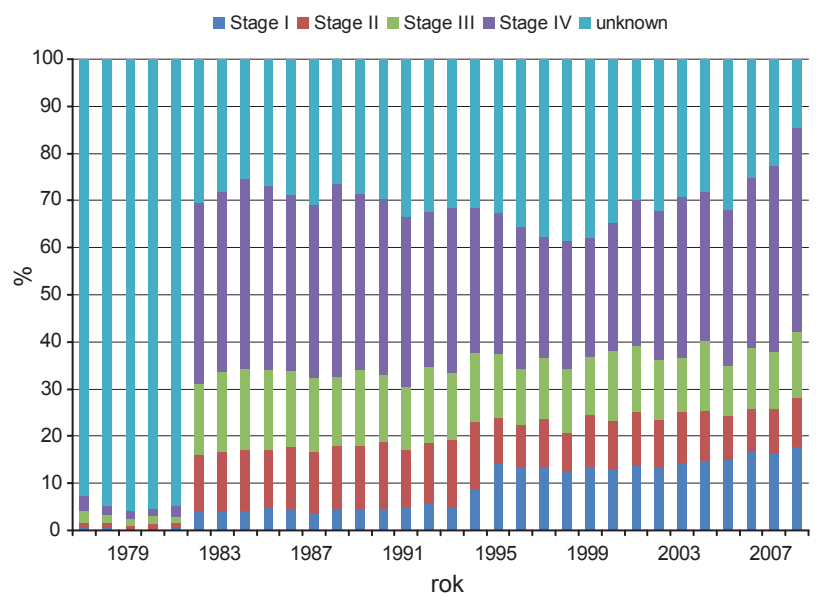

Graph 2. Gastric cancer in the Czech Republic. Trend of distribution of clinical stages.

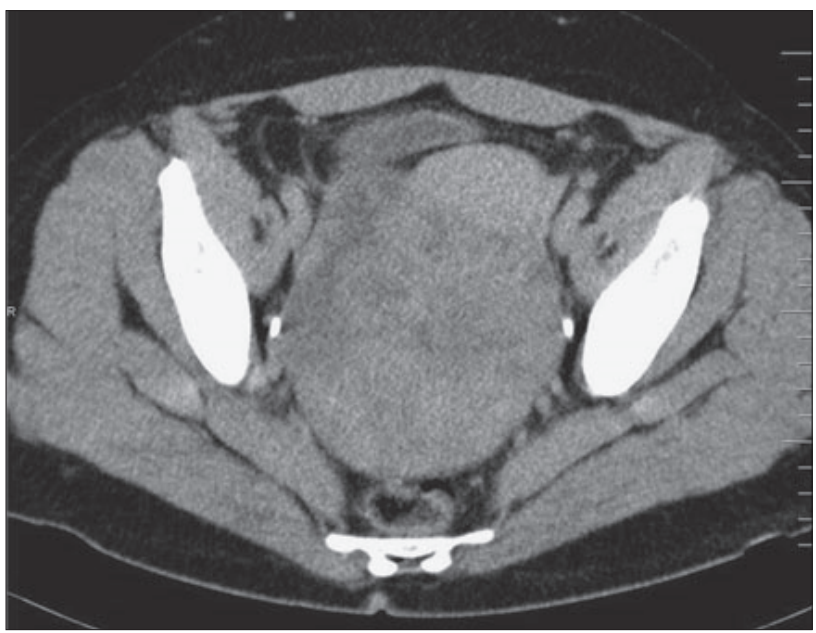

Fig. 1. CT scan with large tumour in small pelvis.

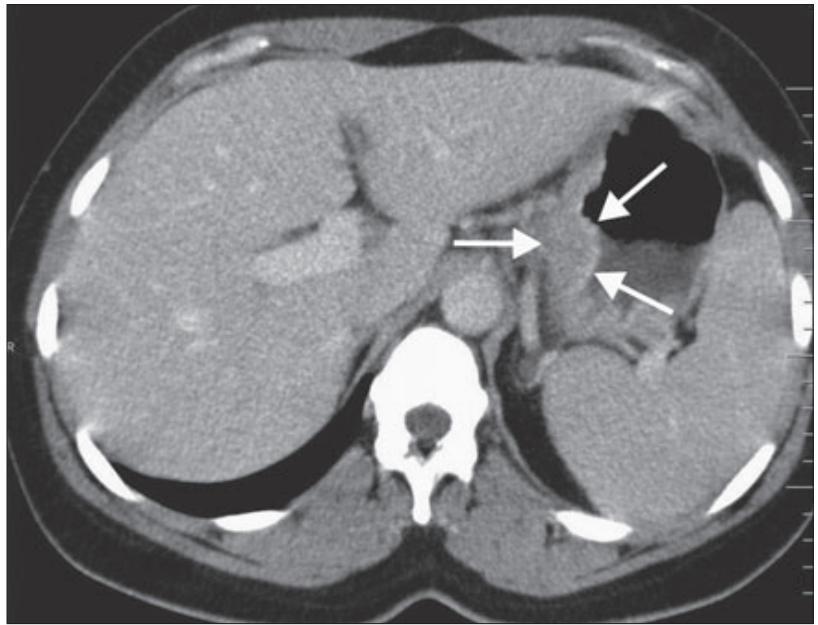

Fig. 2. CT scan before gastrectomy. Arrows indicate the thickening of the gastric wall with tumour.

A 35 years old female was indicated for gynecological surgery due to ovarian tumour (Fig. 1). CT scan before operation

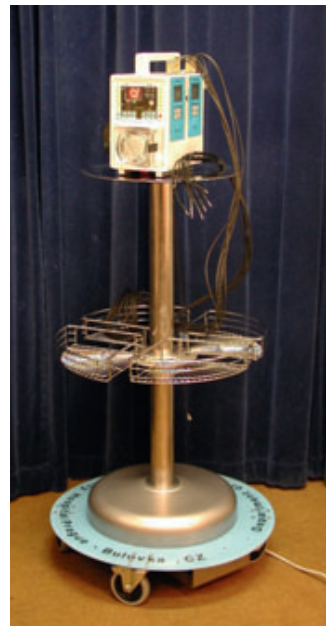

Fig. 3. Original Czech device for HIPEC.

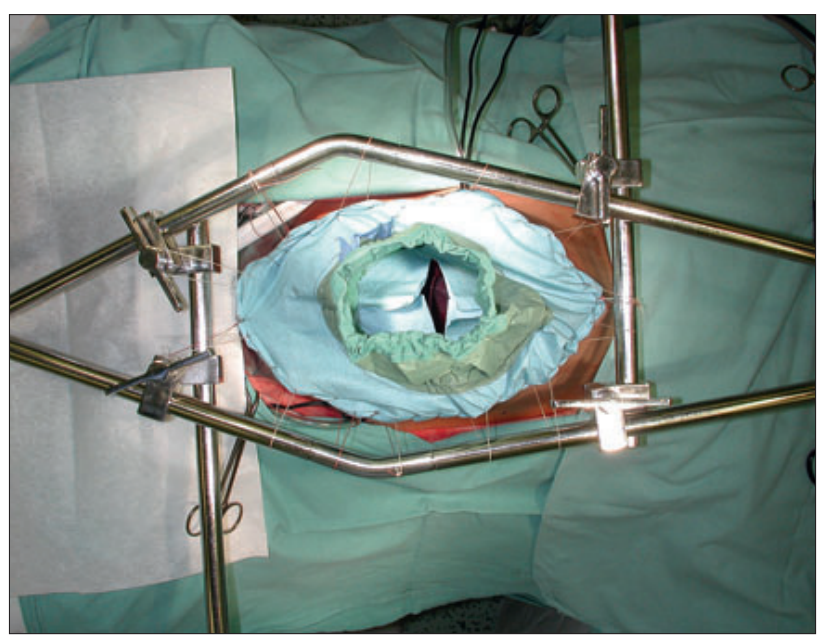

Fig. 4. Coliseum for HIPEC procedure.

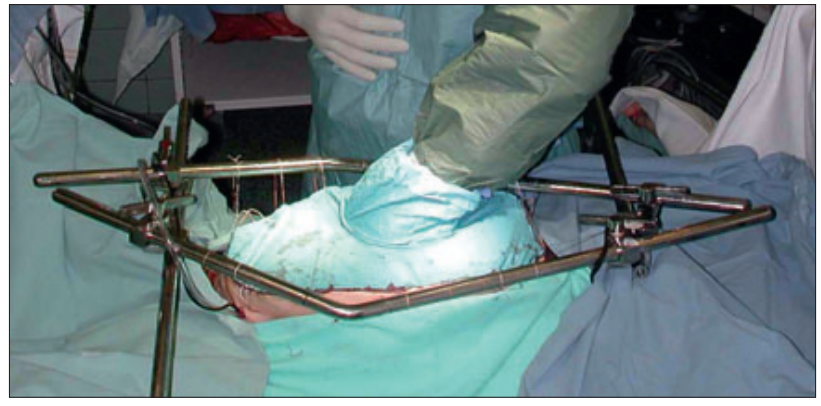

Fig. 5. Open HIPEC procedure.

did not find any other pathology. However, this scan was focused only on small pelvis. Hysterectomy and bilateral adnexectomy was performed. The result of the peroperative patological examination was surprisingly not ovarian tumour but a metastasis of the gastrointestinal tumour (most probably gastric tumour). Apendectomy was performed as well and there was a metastasis too. Surgeon was consulted during the operation and the decision was to finish the operation and after that the examination of the 
patient and if the gastric cancer would be confirmed, to perform gastrectomy + HIPEC.

Gastroscopy found a diffuse gastric cancer and new surgery was indicated 1 month later. The levels of the tumour markers before operation were normal (CEA 0, $9 \mu \mathrm{g} / 1$ and $\mathrm{Ca} 19-91,1 \mathrm{U} /$ $/ \mathrm{ml}$ ). CT scan focused on stomach confirmed tumour, too (Fig. 2).

\section{Surgery}

Stomach body was infiltrated with tumour, but prepyloric region and esophagus was without macroscopic signs of tumour expansion. There were no signs of liver and peritoneal spread. The peritoneal lavage for cytology and biochemistry examination was performed. The tumour was fixed to the mesotransversum, from where it was possible to detach. A total gastrectomy with D2 lyphadenectomy was performed. The duodenal and esophageal stumps were closed with linear staplers. Peroperative examination of the lymph nodes in the spleen hilus was negative, so splenectomy was not performed. The fatty tissue from mesotransversum was excised and a part of the scar after gynecologic operation was excised too. Peroperative hypertermic cytostatic lavage was performed (90 minutes, $42 \mathrm{C}$, Doxorubicine $90 \mathrm{mg}$ ) (Figs 3-5). Esophagus - Roux $\mathrm{Y}$ - jejunostomy without pouch was used for reconstruction. There was postoperative isothermic cytostatic lavage for 5 postoperative days (Fluorouracil $1000 \mathrm{mg} /$ day). An elevated temperature occurred on the 10th postoperative day and fluid collection was diagnosed under the left diaphragm. The CT navigated drainage successfully solved this collection.

Result of pathologic, cytological and biochemistry examination

Stomach - gastric carcinoma, diffuse type, infiltrating the whole gastric wall and omental fat, too.

Lymph nodes -21 nodes, 9 of them with metastases

Part of the mesotransversum and gynecologic scar - infiltration with cancer cells. Cytoceratine $\mathrm{AE} 1 / 3$ positive

Cytological examination - without malignant cells, mesotelial activation

Biochemistry ofabdominal lavage-CEA $0,8 \mu \mathrm{g} / 1, \mathrm{Ca} 19-9$ 2,2U/1

TNM - pT3N2M1, stage IV

Follow up

The palliative chemotherapy was given postoperatively and the patient was regularly checked every 3 months. The clinical examination, laboratory test, endoscopic examination and CT or PET CT were always performed. Patient was without clinical sign of recurrence, with normal levels of CEA a Ca 19-9 and normal endoscopic examination. PET CT showed glucose accumulation in upper part of the abdomen one year later. The second look surgery was indicated.

\section{2nd surgery}

The opening of the abdomen was difficult due to many adhesions. There was diffuse peritoneal infiltration with tumour. Tumour nodules were on average about $3 \mathrm{~mm}$ large and both parietal and visceral surface was involved. There were large nodules about $3 \mathrm{~cm}$ in diameter in upper and lower part of abdomen. Gilly peri-

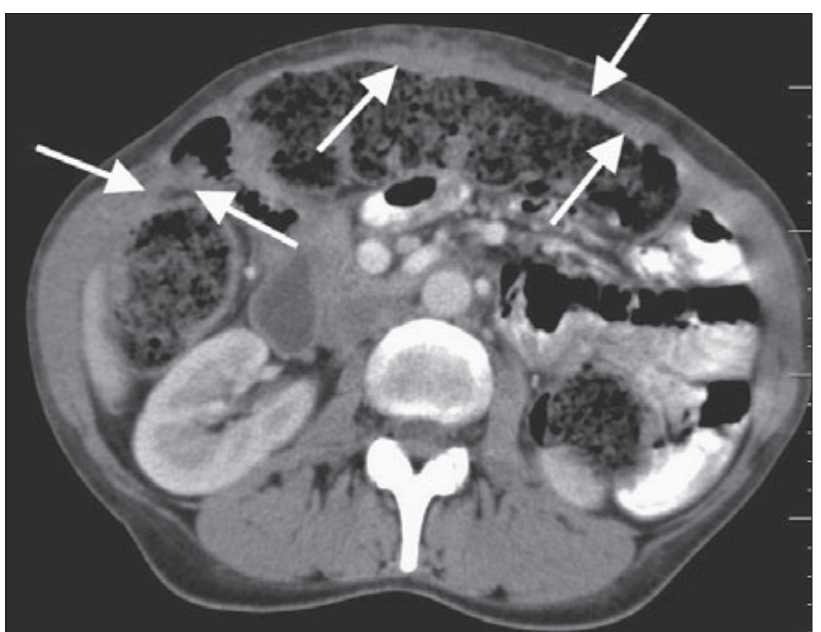

Fig. 6. CT scan 6 month after second HIPEC procedure. Arrows indicate peritoneal tumour seeding.

toneal index was 4 (Tab. 1). Lavage for cytological and biochemistry examination was done and only hypertermic intraoperative cytostatic lavage was performed in the same manner, like during the first surgery.

Pathologic examination from peritoneum confirmed diffuse gastric cancer $\mathrm{AE} 1 / 3$ positive. Cytological examination was again without malignant cells but CEA was $157,9 \mu \mathrm{g} / 1$ and Ca 19-9 was $7688 \mathrm{U} / 1$.

\section{Follow up}

Patient was treated with palliative chemotherapy. She was regularly checked only by clinical examination. She died 18 month after the second surgery. The ascites was not present and there were no signs of intestinal obstruction (Fig. 6). Only anemia developed several months before her death probably due to occult GI bleeding or bone marrow infiltration.

Multiple liver, lymph nodes (incl. groin nodes and mediastinal nodes), pancreatic and peritoneal metastases were found during necropsy.

Tab. 1. Gilly peritoneal carcinomatosis staging system.

\begin{tabular}{|c|c|}
\hline Stage & Peritoneal carcinomatosis description \\
\hline Stage 0 & No macroscopic disease \\
\hline Stage 1 & $\begin{array}{l}\text { Malignant implants less than } 5 \mathrm{~mm} \text { in diameter localized in one } \\
\text { part of the abdomen }\end{array}$ \\
\hline Stage 2 & $\begin{array}{l}\text { Malignant implants less than } 5 \mathrm{~mm} \text { in diameter diffuse to the } \\
\text { whole abdomen }\end{array}$ \\
\hline Stage 3 & Malignant implants $5 \mathrm{~mm}$ to $2 \mathrm{~cm}$ \\
\hline Stage 4 & Large malignant nodules (more than $2 \mathrm{~cm}$ ) \\
\hline
\end{tabular}

Tab. 2. Completeness of cytoreduction (CCR).

\begin{tabular}{ll}
\hline CCR score & Description \\
\hline CCR-0 & no macroscopic nodule is visible at the end of cytoreduction; \\
CCR-1 & residual microscopic nodules $<2.5 \mathrm{~mm}$ \\
CCR-2 & residual nodules between $2.5 \mathrm{~mm}$ and $2.5 \mathrm{~cm}$ \\
CCR-3 & residual macronodules $>2.5 \mathrm{~cm}$ \\
\hline
\end{tabular}




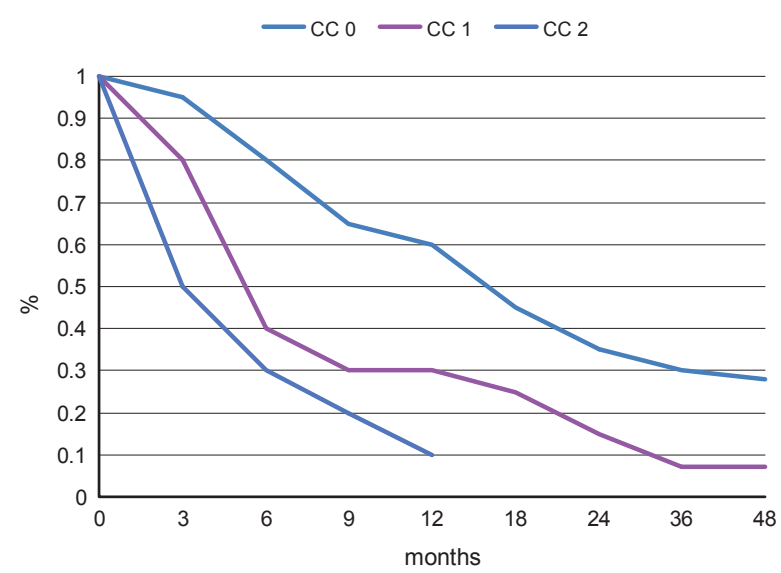

Graph 3. Overall survival according to completeness of cytoreductive surgery.

Patient with metastatic diffuse gastric carcinoma treated by radical surgery with HIPEC procedure in combination with oncologic chemotherapy survived 32 months after diagnosis.

\section{Discussion}

Hyperthermic intraoperative intraperitonel chemotherapy (HIPEC) is relatively new surgical technique. HIPEC is based on Sugerbaker's conception - peritoneal tumour spread is similar process like hematogenic and lymphogenic dissemination. Advanced surgical resection including peritonectomy in addition with intraoperative chemotherapy is the key of the procedure. Mitomycin $\mathrm{C}$, Doxorubicin and carboxyl or cisplatine are the most used chemotherapeutic agents $(7,8)$.

HIPEC procedure is contemporary indicated (in experimental studies) for these diagnoses $(9,10)$ :

- Pseudomyxoma peritonei,

- Peritoneal mesotelioma,

- Peritoneal metastasis due to GI cancer,

- Perforated GI cancers,

- Cancer infiltrating surrounding organs,

- GI cancers with positive peritoneal cytology,

- Malignant ascites (palliative treatment).

There are published contemporary studies about HIPEC in the treatment of the advanced gastric carcinoma. These studies confirmed the potential of this treatment. In the case of a complete cytoreduction (Tab. 2), the survival is significantly longer $(11,12)$. The results are influenced by the extent of the disease (only T3 or T4 versus Gilly 1 or 2 versus Gilly 3 or 4). On the other hand, Hall did not confirm the superiority of HIPEC (13). The metaanalysis (2004) assess 11 studies with 1161 patients (14). The result of this metaanalysis was that HIPEC could improve the survival of the patients with advanced gastric cancer. The metanalysis from 2007 with 13 studies and 1648 patients confirmed better treatment results with HIPEC technique alternatively in addition with early postoperative intraperitoneal chemotherapy (EPIC) (15).

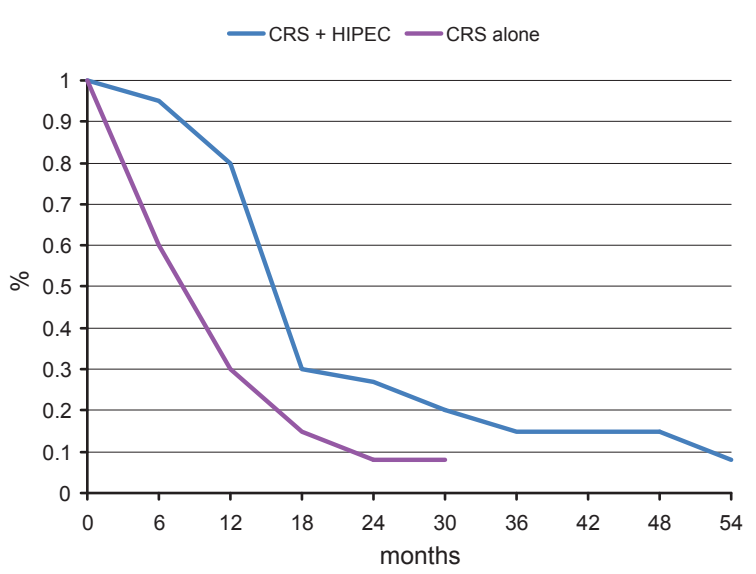

Graph 4. Survival after cytoreductive surgery with or without HIPEC.

The occurrence of complications and mortality rate was also observed in all studies. Yan detected a higher risk of intraabdominal abscesses and higher risk of neutropenia in the group with HIPEC (15). Zhu presented a higher risk of postoperative complication but the difference was not statically significant (23.08 : $12.12 \%$ ). Renal failure was the most observed complication. There was not any death related to the surgery (12). 30-day mortality about 5.4 $\%$ and long term mortality $11 \%$ was presented in another study. GI fistulas occurred most frequently (11). Hall found a higher complication rate in the group with HIPEC (35\% vs $17 \%$ ) but the mortality rate was higher in the group without HIPEC (1 \% vs $0 \%$ (13). Glockin published morbidity after cytoreductive surgery $25-41 \%$ and mortality $0-8 \%$. There were not only patients suffering from gastric carcinoma. He divided the complication into the two groups (16):

- Surgical complication (wound infection, anastomotic leak, ileus etc.),

- Complication related to chemotherapy (leucopenia, thrombocytopenia, anemia, liver toxicity, kidney toxicity etc.).

Similar results were published by Glehen (17) (Graph 3 and 4).

\section{Conclusion}

Aggressive surgical resection in addition with HIPEC and EPIC is a new treatment option, which could improve the treatment result in the cases of advanced gastric carcinoma, especially in patients in the risk of peritoneal spread (T3-T4) or with peritoneal seeding (Gilly 1-2). A completeness of the cytoreduction $(\mathrm{CC} 0-\mathrm{CC} 2)$ influences the prognosis. This technique could improve the survival rate and the quality of life of inoperable patients

\section{References}

1. EUNE for Gastric Cancer Steering Group: Gastric cancer in Europe. Brit J Surg 2008; 95: 406-408. 
2. Roth AD. Curative treatment of gastric cancer: towards a multidisciplinary approach? Crit Rev Oncol Hematol 2003; 46: 59-100.

\section{3. http://swod.cz}

4. JCGA: Introduction to JGCA gastric cancer treatment guidelines. http://'W'VoIw .j gca.jp/PDF files/E-gudeline.PD F.

5. Becker HD, Hohenberger W, Junginger T, Schla PM. Chirurgická onkologie. Praha: Grada Publishing a.s., 2005.

6. Townsend CM Jr et al. Sabiston Textbook of Surgery. 17th ed. Elsevier 2004.

7. Sugerbaker PH. Management of peritoneal surface malignancy using intraperitoneal chemotherapy and cytoreductive surgery. Washington cancer institute 1998 .

8. Sugerbaker PH. Technical Handbook for integration of Cytoreductive Surgery and Perioperative Intraperitoneal Chemotherapy into the Surgical Management of Gastrointestinal and Gynecologic Malignancy, Washington cancer institute, 2005.

9. Deraco M. Cytoreductive Surgery and Intraperitoneal Hyperthermic Perfusion. Eur Oncol Dis 2007. http://w.vvw.touchbriefings.com/pdfl2 7 89/Deraco.pdf.

\section{0. www.surgicaloncology.com}

11. Scaringi $S$ et al. Advanced gastric cancer with or without peritoneal carcinomatosis treated with hyperthermic intraperitoneal chemotherapy: A single western center experience. EJSO 2008; 34: 1246-1252.
12. Zhu ZG et al. Efficacy and Safety of Intraoperative Peritoneal Hyperthermic Chemotherapy for Advanced Gastric Cancer Patiens with Serosa Invasion. Dig Surg 2006; 23: 93-102.

13. Hall J et al. Cytoreductive Surgery With Intraperitoneal Hyperthermic Chemotherapy for Advanced Gastric cancer. J Gastrointest Surg 2004; 8: $454-463$.

14. Xu DZ, Zhan YQ, Sun XW et al. Meta-analysis of intraperitoneal chemotherapy for gastric cancer. World J Gastroenterol 2004; 10 (18): 2727-2730.

15. Yan TD et al. A Systematic Review and Meta-analysis of the Randomized Controlled Trials on Adjutant Intraperitoneal Chemotherapy for Resectable Gastric Cancer. Ann Surg Oncol 2000; 14 (10): 2702-2713.

16. Glockzin G, Schlitt JH, Piso P. Peritonea carcinomatosis: patiens selection, perioperative complications and duality of life related to cytoreductive surgery and hyperthermic intraperitoneal chemotherapy. World J Surg Oncol 2009; 7: 5.

17. Glehen $O$ et al. Peritoneal Carcinomatosis from Gastric Cancer: A Multi-Institutional Study of 159 Patients Treated by Cytoreductive Surgery Combined with Perioperative Intraperitoneal Chemotherapy. Ann Surg Oncol 2010; 17: 2370-2377.

18. Xiao-Jun Yang, Chao-Qun Huang, Tao Suo, Lie-Jun Mei, GuoLiang Yang et al. Cytoreductive Surgery and Hyperthermic Intraperitoneal Chemotherapy Improves Survival of Patients with Peritoneal Carcinomatosis from Gastric Cancer: Final Results of a Phase III Randomized Clinical Trial. Ann Surg Oncol 2011; 18: 1575-1581. 